and the family history was unimportant. The patient's general health was good. He had had a suspicious luetic lesion twenty-four years ago.

Visual Examination.-Visual acuity: O.D., 4/60; O.S., 6/60. Lids, lacrimal apparatus, conjunctiva and cornea appeared healthy. Rotations of the muscles were good; there was no history of diplopia, and none was elicited with the colored glass. Pupils: right, $4.5 \mathrm{~mm}$; left, $4 \mathrm{~mm}$.; no reaction to light, direct or consensual. Pupillary (Wernicke's) inaction test absent. Both eyes showed a sluggish reaction to accommodation. Fundus: right media was clear; disk pale, reniform, axis at 90, lamina not visible and cupping shallow, excessive heaping of pigment along rasal border and moderate pigmentation at the lower temporal side; the general outline was well defined; the retinal vessels presented a healthy, normal appearance. Left eye a general haze of lens, vitreous clear; disk slightly oval at 90 , outline well defined with a moderate pigment border, color pale, lamina not visible, cupping shallow. Vessels appeared healthy. The general fundus picture of each eye, with the exception of the atrophic color of the disk, was normal.

Visual Fields: The first field was mapped April 4 and repeated testing disclosed a well-defined nasal defect. At the end of the second week the patient was accepted as a house case of another institution and remained there for nine weeks. He returned to our observation June 27 , with a vision equal to hand movements in the right eye, left eye 2/60. Candle

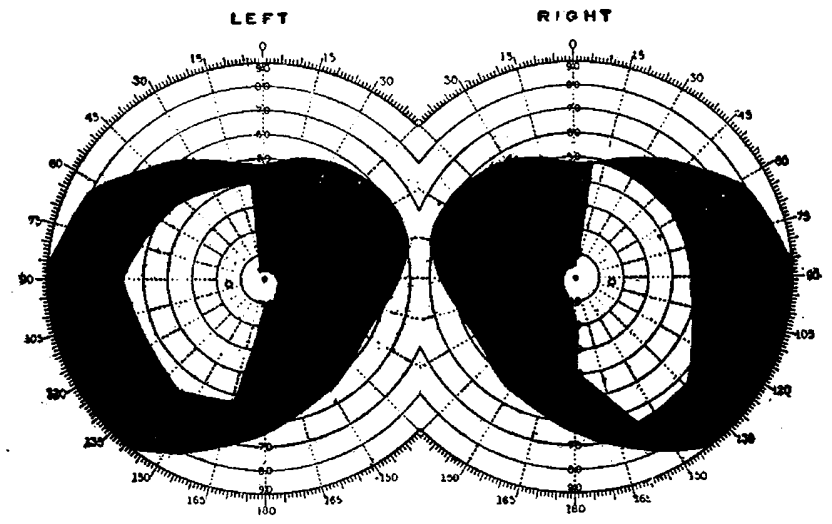

Chart 2.-Perimetric candle fleld, Aug. 5, 1912.

fields at this time showed a clear-cut binasal defect. Both optic papillae presented a decided atrophic pallor.

Neurologic Examination.-The gait was normal; Romberg's sign was present, but not very marked. There was no involvement of the cranial nerves other than the optic. Grip with both hands was strong; there was no ataxia of the upper extremities, Biernacki's sign was present. There were no muscular abnormalities, no trophic changes or sphincter disturbanes. General sensibility was normal, with no astere ognosis. Knee-jerks were normal; the Achilles tendon reflex was lost, both sides. There were no Babinski sign or ankle clonus, no girdle sensation, lightning pains, visceral crises or loss of sexual power. Iysterical stigmata were absent.

Examination of the cerebrospinal fluid by Dr. E..D. Funk showed a lymphocytosis.

A Wassermann test, made by Dr. George F. Lull, was nega tive, which in all probability was due to the patient having been under mercurial treatment before the test was made. Since being under our observation the patient has taken potassium iodid freely, and had eighteen inunctions of mercurial ointment ( 1 dram, each) before having the slightest tenderness of the gums, so that the therapeutic test may be said to have been positive. The urinary findings were negative.

A neurologic diagnosis of tabes was made. We consider this warranted by the combination of optic atrophy, slight Romberg sign, Biernacki's sign, loss of the Achilles tendon reflex, and a lymphocytosis of the cerebrospinal fluid.
The binasal defect in the visual fields we believe to be the result of a selective atrophy affecting the intracranial portion of the optic nerves.

1700 Walnut Street.

\section{CASE OF STRANGULATED TUBO-OVARIAN HERNIA IN AN INFANT *}

Arthur B, Eustace, M.D., and R. William McNealy, M.D., CHICAGO

In a contribution of recent date Heineck ${ }^{\mathbf{1}}$ presented an analytic review of all undoubted cases of tubo-ovarian hernia reported in the French, German and English medical literature from 1890 to 1910 , inclusive.

He cited eighty cases of hernia of this type, in which thirty-five occurred in infants under 1 year of age. The large majority of those hernias occurring in infants were irreducible and showed evidence of strangulation at operation. There were only two fatalities reported from these thirty-five cases, although in four no end-results were given.

All hernias of this type occurring in children were in the inguinal region and femoral hernias in adults only, with special reference to this condition.

History.-A colored infant, aged 6 months, brought to our clinic Wednesday morning, had always been in perfect health except for hernia. The infant was breast-fed. The Sunday night previous the mother noticed that the "rupture" (which she had noticed soon after the child was born) had come down argain following or during a severe crying-spell. She attempted to reduce it as she had been in the habit of doing many times before, but found that she was unable to do so.

The following day the child became noticeably restless and was constantly fretful and was taken to the family physician, who told the mother to apply hot applications to the tumor mass and return on the following day.

On Tuesday the external swelling had noticeably increased in size and showed evidence of inflammation, whereupon the mother took the child to another physician, who gave the child some "drops" and advised more hot applications to the swelling. During the night the child vomited twice and had a scant bowel movement.

Examination.-The infant, which appears well nourished and quite active, does not seem to be in any great distress. The abdomen is slightly distended. The right labium majus is swollen, red and very tender, pits on pressure and has a translucent appearance. The tumor mass is about the size of an English walnut. It is impossible to elicit either fluctation or pulsation. There is no evidence of vaginal discharge. The temperature is $103.4 \mathrm{~F}$.

After a compilation of the data a diagnosis of strangulated inguinal hernia was made and immediate operation advised. operation.-The infant was given a preliminary colonic flushing. Under ether anesthesia a regular right inguinal incision was made, the inguinal canal exposed and the hernial sac delivered into the field of operation. The peritoneum was of a dark-brown chocolate color and the small vessels appeared to be thrombosed. The sac was opened and about 2 drams of bloody fluid escaped, exposing a black congested mass which was not immediately recognized. With gentle traction this mass was drawn down into the inguinal canal, whereupon it was found to be a tube, ovary and broad ligament. The right cornu of the uterus could easily be brought into the canal and the tubal attachment demonstrated. There was a torsion of the tube and ovary in a clockwise direction of about 180 degrees. The tube and ovary were immediately resected and the stump returned to the abdomen. The peritoneal sac was freed about the hernial ring, transfixed and ligated. The Andrews imbrication method was used to close the parietes. The skin was closed with running silkworm-gut and no drainage used. Gutta-percha and adhesive protection of wound was employed.

* From their surgical clinic at the Post-Graduate Hospital, Chicago.

1 Surg., Gynec. and Obst., $x v$, No. 1, p. 63. 
The child was put to the breast twelve hours after the operation. She remained in the hospital for twelve days.

Pathologic Report.-Examination of a specimen of the tube and ovary revealed that the tube was edematous and showed necrotic areas. The blood-vessels were thrombosed and there was extravasated blood in the lumen. The ovary was gangrenous.

The excellent prognosis in these cases stands out in contradistinction to strangulations in which the intestines are involved.

4238 Indiana Avenue.-4254 Indiana Avenue.

GONORRHEAL INVOLVEMENT OF THE SKIN OF THE PREPUCE CURED BY UNUSUAL THERAPEUSIS *

\section{ZigLer, M.D., New YoRK}

Instructor in Genito-Urinary and Venereal Diseases in the PostGraduate Medical School and Hospital, Chief of Clinic in Genito-Urinary Lebanon Hospital, Assistant Dermatologist Lebanon Hospital, Out-Patient Department

As most authorities assert such an involvement rarely if ever occurs, I report this case of a low-grade inflammation of the skin of the prepuce, due to gonorrhea.

History.-Wm. S., aged 20, contracted gonorrhea three years ago. Three weeks after the first attack he noticed an area of redness on the under surface of the prepuce and that on pressure pus exuded from several points. Numerous antiseptic solutions were used to heal this lesion with negative result. Escharotics were tried without success. After several unsuccessful alcempts to induce the infiammatory process to heal by other means, the surgeon in charge laid it open by a longitudinal incision through its entire length. Two months after operation the affected area was slightly improved; that is, it was still red and inflamed but there was no oozing of pus and only an occasional watery (serous) discharge, which continued until about one month before he came under my observation, when it had again become purulent. The patient says that "on pressure drops of pus can be expressed from several points in the inflamed area."

Physical Examination.-In April, 1913, examination of the patient showed a long prepuce on the anterior surface of which, one-half inch from the tip, extending in the long axis of the penis, was a linear lesion about 1 inch in Iength, and a quarter of an inch in width, somewhat raised above the level of the surrounding skin, reddish and which felt like a cord or a thread under the skin. Yellowish-white pus could be expressed from three distinct points, one of which was at the tip near the distal end of the penis, the second in the middle of the lesion, and the third at the proximal end. When probed the points were found to be superficial.

The pus was examined microseopically by Dr. R. M. Taylor of the pathologic department of the Post Graduate Medical School (N. Y.). He reported it as composed of polynuclear leukocytes, a considerable number of which contained Gramnegative cocci, typical of the gonococci in their morphologic characteristics and arrangement within the cell.

Culture Growths.-Plates on rabbit-blood agar and ascitic fluid agar, showed, after twenty-four-hour incubation, numerous small grayish-white colonies which on microscopic examination proved to be Gram-negative cocei. Transfers from these colonies on ascitic agar grew with the characteristic appearance of the gonococcus. Therefore microscopically and culturally the organism was assumed to be a gonococcus.

Complement-Fixation Test.-The organism was subsequently identified as the gonococcus by the complement-fixation test.

Treatment and Course.-After the receipt of the pathologic report, I used a strong antiseptic wash of full strength hydrogen peroxid locally and later mercuric chlorid solution $1: 1,000$ for several weeks, but the lesion failed to heal. I then decided to eircumcise, but on careful examination of the lesion I found that it extended backward beyond the corona and was therefore afraid that so much of the prepuce would

* Reported at the Bronx Medical Society, September, 1913, at the Lebanon Alumni Society, May, 1913. have to be removed that later a curvature of the penis might occur during erection. I therefore decided on a more conservative plastic operation.

Operation and Result.-April 25, 1913, I excised an oval piece of the skin of the prepuce about 1 inch long extending from one-third of an inch from the tip of the prepuce to onethird of an inch back of the corona. The edges of the wound were brought together by four silkworm sutures. The wound healed by primary union in one week. When the patient was seen for the last time Sept. 7, 1913, no vestige of the lesion could be seen.

Pathologic Examination.-The excised tissue was examinea by Dr. R. M. Taylor, who made the following report:

The specimen is an oval-shaped elongated piece of skin slightly flattened on one side. About $4 \mathrm{~mm}$. from one end is seen an opening through the skin surface which leads into a sinus that can be followed through the middle of the tissue and ends blindly. The microscopic picture shows a sinus surrounded immediately beneath its epithelial layer by a mild acute exudative inflammation extending deeply into the subepithelial tissue where it is limited by a band of productive inflammation, as shown by the presence of young granulation tissue. Careful search for organisms (gonococei) by means of special strains was negative in result. This case is reported because of the rarity of occurrence of cutaneous gonorrhea and the unuisual therapeutic procedure.

1097 Forest Avenue.

\section{IDIOSYNCRASY TO ASPIRIN (ACETYLSALICYLIC ACID)}

Edward N. Reed, M.D., Clifton, Ariz.

The patient, Mr. T. W. T., American, aged 36, elerk in a store, had the diseases of childhood and had typhoid at the age of 19 ; otherwise he has never had any serious sickness.

January 29 , at 7 p. m., he took one capsule containing .; grains of aspirin, for a "cold." In about half an hour after taking the capsule he vomited, and in another fifteen minutes, he says, "his throat felt stiff and dragged when he swallowed, and he thought he was developing tonsillitis." In an hour and a half after taking the capsule he had reached the serious con dition in which I found him at $9: 30$, two and a half hours after taking the medicine. At that time the face was cyanotic, the lips and ears violaceous in color, the eyelids so edematous as to be swollen almost shut, and conjunctivae much injected. The whole face was swollen. The breathing was extremely labored, like asthmatic breathing. The nasal mucous mem. brane was so engorged that breathing through the nose was impossible. The patient had vomited several times. Inspec tions of the mouth showed the buccal mucus membrane, fauces and pharynx dark red and swollen. The uvula was swollen to twice its normal size. The rest of the body was normal in appearance.

The pulse was 120, soft and full, the temperature 98. Auscultation of the heart was negative. In the lungs were numerous dry whistling râles, but no moist ones. The breathing was such as one would expect with an edema of the glottis. The urine was negative.

No treatment was instituted. In about six hours the patient was comfortable, the nose patulous and the breathing normal. The following morning there was a fine discrete papular rash on the trunk.

The patient reports that he had a "cold" about a year ago, for which he took one capsule, given him by a druggist (which I have ascertained to have contained aspirin and acetphe. netidin, of each $2 \frac{1}{2}$ grains ), and that a condition similar to the one here reported resulted, lasting about five hours.

Meaning of Public Health.-An individual cannot live economically, socially, mentally or morally unless he has health. Neither can a nation. Until people can be made to see this viewpoint, public health will mean nothing more than a placard and a bottle of formaldehyd.-William DeKleine in Puhlic Health (Mich.). 